\title{
Exeter Book riddle titles
}

For explanation, see the General Introduction that follows this list. For a full discussion of these titles, see Jennifer Neville, 'A Modest Proposal: Titles for the Exeter Book Riddles', MAE 88 (2019), 116-23.

$\begin{array}{lll}\text { Riddle title } & \text { Title translation } & \text { ASPR }\end{array}$ riddle no.

\begin{tabular}{|c|c|c|}
\hline Drymful Punie & 'Glorious I Thunder' & 1 \\
\hline Under Ypa Geprcec & 'Under the Force of Waves' & 2 \\
\hline Min Frea Faste Genearwad & $\begin{array}{l}\text { 'My Lord Constrains Me } \\
\text { Tightly' }\end{array}$ & 3 \\
\hline Drymful Peow & 'Glorious Servant' & $\begin{array}{l}1-3 \text { as } \\
\text { one riddle }\end{array}$ \\
\hline Garsecges Grund & 'Ocean's Bottom' & $\begin{array}{l}2-3 \text { as } \\
\text { one riddle }\end{array}$ \\
\hline Dragbysig & 'Periodically Busy (?)' & 4 \\
\hline Anhaga & 'Lone Dweller' & 5 \\
\hline Ic Cwice Barne & 'I Burn the Living' & 6 \\
\hline Ferende Gaest & 'A Travelling Spirit' & 7 \\
\hline Eald AEfensceop & 'Old Evening Poet' & 8 \\
\hline Mec Deadne Ofgeafun & 'They Gave Me up for Dead' & 9 \\
\hline Neb Wees Min on Nearwe & 'My Nose was in Confinement' & 10 \\
\hline Hasofag & 'Grey (?)' & 11 \\
\hline Fotum Ic Fere & 'I Travel on Foot' & 12 \\
\hline$X$ Gebropor ond Sweostor & 'Ten Brothers and Sisters' & 13 \\
\hline Wrepenwiga & 'Weapon-Warrior' & 14 \\
\hline Hals Hwit ond Heafod Fealo & 'White Neck and Fallow Head' & 15 \\
\hline Wib Wage Winnan & 'Fight against a Wave' & 16 \\
\hline Mundbora & 'Protector' & 17 \\
\hline Wide Wombe & 'Large Womb' & 18 \\
\hline Heafodbeorht & 'Bright-headed' & 19 \\
\hline Wrepnum Awyrged & 'Cursed among Weapons' & 20 \\
\hline Neol ic Fere & 'I Go Down' & 21 \\
\hline
\end{tabular}


Riddle title

Fridhengestas

Agof

Siex Stafas Sweotule Becnap

Stapol min is Steapheah

Nama Min is Mare

Bindere ond Swingere

Dream bið in Innan

Lyftfat Leohtlic

Legbysig

Ligbysig

Fepegeorn

Grindan wið Greote

Gryrelic Hleahtor

Seo pat Feoh Feded

Hyhtlic Gewad

Monn Wiif Hors

Siex Heafdu

For Flodwegas

Womb waes on Hindan

Geoguðmyrpe Grcedig

Earmost Ealra Wihta

Ymbhwyrft

Ymbhwyrft Edniwu

Moddor Monigra Cynna

Heanmode Twa

Giest in Geardum

Wratlic Hongad

Banleas

Wer Sat at Wine

Moððе

Hring butan Tungan

Eardfast

Torht Wiga

Wuhte Feower

Rapingas

Beam Hlifian

In Wincsele

Wulfheafedtreo

Holt Hweorfende
Title translation

ASPR

riddle no.

22

'Peace-horses (?)'

22

'Wob' ('bow' misspelled

23

backwards)

'Six Letters Clearly Show' 24

'My Foundation is Steep and 25

High'

'My Name is Famous' 26

'Binder and Scourger' 27

'Joy is Inside' 28

'Bright Air-Vessel' 29

'Occupied with Flame' 30a

'Occupied with Flame' 30b

'Eager to Go' 31

'Grind Against Grit' $\quad 32$

'Terrible Laughter' 33

'The One who Feeds Cattle' 34

'Hopeful Garment' 35

'Man, Woman, Horse' 36

'Six Heads' 36.1-7

'Travel the Flood-ways' 36.8-13

'Womb was Behind' 37

'Greedy for Youth-Mirth' 38

'Most Wretched of All 39

Creatures'

'Circle of the Earth' $\quad 40$

'Circle Renewed' 40-41 as

one riddle

'Mother of Many Races' 41

'Two Low-Minded Ones' 42

'Guest in the Enclosures' 43

'A Wonder Hangs' 44

'Boneless' 45

'A Man Sat at Wine' 46

'Moth' 47

'Ring without a Tongue' $\quad 48$

'Earth-fast' 49

'Bright Warrior' 50

'Four Creatures' 51

'Captives' 52

'Tree Towers Up' 53

'In a Corner' 54

'Wolf-head Tree' 55

'Turning Wood' 56 
Riddle title

Sanges Rope

Anfetu

Hring Gylden

Be Sonde Muðlease

Heafod Sticade

Hingonges Strong

Glad mid Golde

Wynn ond Is

Cwico waes Ic

Mare Donne Middangeard

Wordgaldor

Wiht on Weg Feran

Wundor Weard on Wege

Weter Weard to Bane

Orponcum Geworht

Singeð Durh Sidan

Heah ond Hleotorht

Reade Bewcefed

Mearcpapas Walas Trad

Bragnloca
Feaxhar Cwene
Swift on Swape
Swift ond Sittende
Ides Sittende Ane
Ides Fepeleas

Fepeleas

Orponc Ypum Bewrigene

Apelinges AEht ond Willa

Apelinges Eaxlgestealla

Fyrdrinces Gefara

Byledbreost

Greate Swilgeð

Frod Was Min Fromcynn

Modor Monigra

Nis Min Sele Swige

XII Hund Heafda
Title translation

ASPR

riddle no.

57

'Abounding in Song' 57

'One-footed' $\quad 58$

'Golden Ring' 59

'Mouthless by Sand' 60

'A Head Stuck In' 61

'Strong in Going Out' 62

'Shining with Gold' 63

'Joy and Ice' 64

'I was Alive' 65

'Bigger than the Earth' 66

'Word-Spell' 67

'A Creature Went Away' 68

'A Wonder Happened on the 69

Way'

'Water Turned to Bone' $\quad 68-9$ as

one riddle

'Made with Skill' 70

'Sings through its Sides' 70a

'Tall and Beautiful' 70b

'Clothed in Red' 71

'I Trod the Welsh 72

Boundary-Paths'

'Brain-Locker' 73

'Grey-Haired Queen’ 74

'Swift on a Track' 75

'Swift and Sitting' 75-6 as one riddle

'Woman Sitting Alone' $\quad 76$

'Footless Woman' 76-77 as one riddle

'Footless' 77

'Cunning Thing Covered with 78 Waves'

'Noble's Possession and Desire' 79

'Noble's Shoulder-Companion' 79-80 as one riddle

'Warrior's Companion' 80

'Puff-breasted' 81

'Swallows Grit' 82

'My Ancestry was Old' 83

'Mother of Many' 84

'My Hall is not Silent' 85

'Twelve Hundred Heads' 86 
Riddle title

Title translation

ASPR

riddle no.

Beorcade Wancode

'Barked, Wavered'

87

Broporleas

'Brother-less' 88

Se Wiht Wombe Hafde

'The Creature had a Womb' 89

Lupus

'Wolf'

90

Heafod Homere Gepuren

'Head Forged by a Hammer' 91

Brunra Beot

'Boast of Brown Ones' 92

Gingra Bropor Mec Adraf

'Younger Brother Drove Me 93

Out'

Hyrre ponne Heafon

'Higher than Heaven'

94

Ic Swape Hwilum Mine

'I Sometimes Hide my Track' 95

Bemipe 